\section{The importance of neoplastic cell content assessment and enrichment by macrodissection in cancer pharmacogenetic testing}

Formalin-fixed paraffin embedded samples for actionable somatic pathogenic variant (SPV) testing should undergo review in order to ensure that the appropriate material has been submitted and the neoplastic cell content (NCC) is sufficient for SPV detection. In samples with suboptimal NCC, light microscopy identification of areas of higher NCC and the provision of a marked H\&E slide for precise macrodissection increase the likelihood of detecting an actionable SPV.

While this should reflect common practice, despite requesting this information, our experience is that a significant number of referrals arrive at the laboratory with no indication of NCC and/or are unmarked for macrodissection, necessary on samples with an NCC of less than 10\%. This increases the risk of a false-negative result.

Using malignant melanoma as a representative for actionable SPV testing, we audited 231 samples obtained by the laboratory between August and October 2018. Eight per cent $(n=19)$ of the samples were obtained with no estimate of NCC and $7 \%(n=17)$ of the samples stated to have $<10 \%$ NCC were not marked for macrodissection.

Three exemplar cases of malignant melanoma referred for clinical testing of actionable BRAF SPVs are presented to underline the importance of careful review and identification of neoplastic cell-enriched areas in suboptimal samples.

$B R A F$ testing in malignant melanoma samples is routinely performed using a pyrosequencing assay in this laboratory, which is validated to detect $B R A F$ codon 600 variants down to a level of $10 \%$ variant allele fraction (VAF) reflecting at least 20\% NCC.

Additionally, the Roche cobas 4800 BRAF p.V600 (cobas) assay is used for samples with less than 20\% NCC. This kit is validated to detect the BRAF c. $1799 \mathrm{~T}>\mathrm{Ap}$.V600E variant down to $5 \%$ VAF.

\section{CASE 1}

A wax block from a metastatic melanoma was obtained from a 67-year-old man for BRAF codon 600 testing. No NCC estimate was provided. DNA extracted from unstained whole sections was tested using pyrosequencing and the cobas assay according to locally validated protocols.

Pyrosequencing was suggestive of the BRAF c.1799T >Ap.V600E mutation at an approximate VAF of $2 \%$ (figure $1 \mathrm{~A}$ ), below the validated detection level for this test. The variant was not detected by the cobas assay.

An in-house review of an H\&E stained section identified an area suitable for macrodissection of enriched NCC up to 20\%-30\%. Additional DNA was then extracted from the selected areas.

Subsequently, pyrosequencing detected BRAF c.1799T >Ap.V600E at a reportable VAF of $14 \%$ (figure 1B); a codon 600 variant was also detected by the cobas assay in this sample.

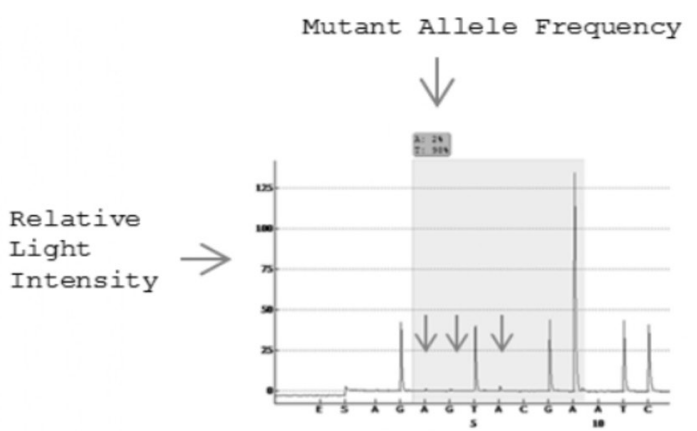

A

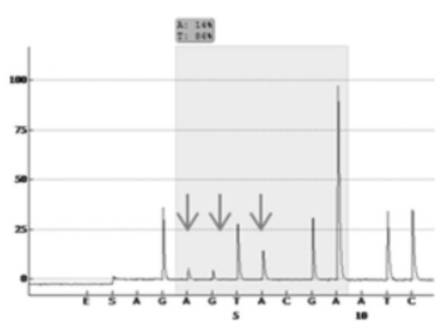

B

Base Dispensation

Figure 1 Case 1 pyrosequencing result. Premacrodissection $(A)$ and postmacrodissection (B) pyrosequencing extension products from the $B R A F \mathrm{c} .1799 \mathrm{~T}>\mathrm{A}$ p.V600E variant are shown (arrows).

\section{CASE 2}

Four unstained slide-mounted sections from a pigmented lesion were obtained from a 45 year old man. The overall NCC was stated to be $10 \%-20 \%$. No H\&E slide was obtained.

Pyrosequencing was suggestive of the BRAF c.1799T>Ap.V600E mutation at an approximate VAF of $2 \%$, below the validated detection level for this test. No codon 600 variants were detected by the cobas assay.

In response to this finding, a marked H\&E slide and further unstained sections were requested and obtained from the referring pathology laboratory. Although the NCC in the marked area was not stated, enrichment by macrodissection resulted in pyrosequencing detecting $B R A F$ c. $1799 \mathrm{~T}>\mathrm{Ap} . \mathrm{V} 600 \mathrm{E}$ at a reportable VAF of $9 \%$, and a codon 600 variant was detected by the cobas assay.

\section{CASE 3}

Slide-mounted paraffin sections from a metastatic melanoma were obtained from a 59-year-old man for BRAF codon 600 testing. The request form indicated an NCC of $10 \%-20 \%$, presumed to be over the whole section. Pyrosequencing and the cobas assay were performed on DNA extracted from the unstained whole sections.

Pyrosequencing was suggestive of the BRAF c.1798 1799delinsAA p.V600K mutation at an approximate VAF of 3\%, but a codon 600 variant was not detected by the cobas assay.

Assessment of an H\&E slide identified two small areas of neoplasia; these were highlighted for macrodissection (figure 2), yielding an NCC greater than $30 \%$. Pyrosequencing then detected $B R A F$ p.V600K at a reportable allele fraction of $5 \%$; a codon 600 variant was also detected by the cobas assay on the macrodissected DNA sample.

Experienced scientists can spot visual cues on a pyrogram that may reflect the presence of a low-level variant in a sample. Steps can then be taken to check and enrich NCC (if possible) and thus confirm the result. Molecular testing for actionable SPVs, however, is progressing to highthroughput next-generation sequencing (NGS)-based techniques, which do not always provide these visual cues. This is already the case in colorectal cancer, which in our laboratory was formerly tested by pyrosequencing but is now tested by NGS. The importance of correct NCC assessment and identification of areas for macrodissection thus becomes even 


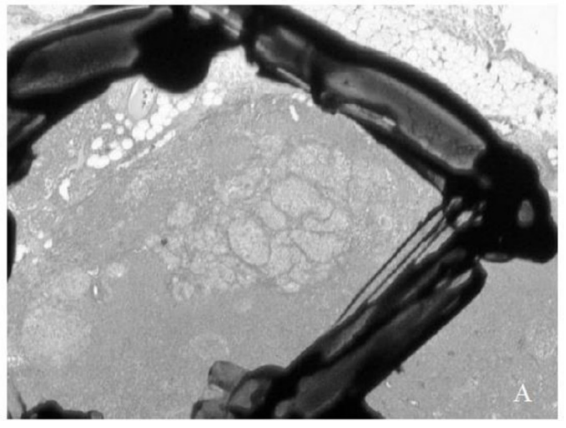

Figure 2 Case 3, metastatic malignant melanoma marked for macrodissection $(\times 2)$.

more important when 'visual' techniques become obsolete.

Microscopic identification of areas of higher NCC and the provision of a marked H\&E slide for precise macrodissection increase the likelihood of detecting an actionable SPV. Overestimation of NCC can result from inclusion of desmoplastic stroma, in addition to the true neoplastic cell population. Additionally, a prominent inflammatory cell population can significantly dilute the level of variant
DNA available and should be taken into account when estimating the percentage of neoplasia in an formalin-fixed paraffin-embedded tissue specimen.

As the outcome of genomic testing increasingly influences clinical management and treatment of cancer patients, ${ }^{1}$ it is of high importance that the adequacy of all samples for genomic testing is assessed by appropriately experienced and competent professionals. Competency assessment for this important function is already available from external quality assessment providers, for example, GenQA (www. genqa.org).

George Joseph Burghel, ${ }^{\oplus 1,2}$ Anne Marie Quinn, ${ }_{1}^{3}$ Philip Smith, ${ }^{\circ}$ Jade Harris, ${ }^{1}$ Catherine Banks, ${ }^{1}$ Andrew Wallace $^{1}$

${ }^{1}$ Manchester Centre for Genomic Medicine, Manchester University NHS Foundation Trust, Manchester, UK

${ }^{2}$ School of Health Science, Faculty of Biology,

Medicine and Health, University of Manchester,

Manchester, UK

${ }^{3}$ Department of Anatomic Pathology, University Hospital Galway, Galway, Ireland

Correspondence to Philip Smith, Manchester Centre for Genomic Medicine, Manchester University NHS
Foundation Trust, Manchester, M13 9WL, UK; philip. smith@mft.nhs.uk

Handling editor Professor Runjan Chetty.

Funding The authors have not declared a specific grant for this research from any funding agency in the public, commercial or not-for-profit sectors.

\section{Competing interests None.}

Patient consent for publication Not required.

Provenance and peer review Not commissioned; internally peer reviewed.

(C) Author(s) (or their employer(s)) 2019. No commercial re-use. See rights and permissions. Published by BMJ.

GJB, AMQ and PS contributed equally.

Check for updates

To cite Burghel GJ, Quinn AM, Smith $P$, et al. J Clin Pathol 2019;72:721-722.

Received 12 July 2019

Accepted 24 July 2019

Published Online First 2 August 2019

J Clin Pathol 2019;72:721-722

doi:10.1136/jclinpath-2019-206094

\section{REFERENCE}

1 Jackson SE, Chester JD. Personalised cancer medicine. Int J Cancer 2015;137:262-6. 